\title{
Dapagliflozin Reduces Adverse Renal and Cardiovascular Events in Patients With Chronic Kidney Disease
}

\author{
Heerspink HJL, Stefánsson BV, Correa-Rotter R, et al. for the DAPA-CKD Trial Committees \\ and Investigators Dapagliflozin in patients with chronic kidney disease. N Engl J Med. \\ 2020;383:1436-1446.
}

\section{Study Overview}

objective. To assess whether dapagliflozin added to guideline-recommended therapies is effective and safe over the long-term to reduce the rate of renal and cardiovascular events in patients across multiple chronic kidney disease (CKD) stages, with and without type 2 diabetes.

Design. The Dapagliflozin and Prevention of Adverse Outcomes in CKD (DAPA-CKD) trial (NCT03036150) was a randomized, double-blind, parallel-group, placebocontrolled, multicenter event-driven, clinical trial sponsored by Astra-Zeneca. It was conducted at 386 sites in 21 countries from February 2, 2017, to June 12, 2020. A recruitment period of 24 months and a total study duration of 45 months were initially planned. The primary efficacy analysis was based on the intention-to-treat population. This was the first randomized controlled trial designed to assess the effects of sodium-glucose co-transporter 2 (SGLT2) inhibitors on renal and cardiovascular outcomes in patients with CKD.

Setting and participants. This trial randomly assigned 4304 adult participants with CKD stages 2 to 4 (an estimated glomerular filtration rate [GFR] of 25 to $75 \mathrm{~mL} / \mathrm{min} / 1.73 \mathrm{~m}^{2}$ of body-surface area) and elevated urinary albumin excretion (urinary albumin-to-creatinine ratio of 200 to 5000 , measured in mg of albumin per $\mathrm{g}$ of creatinine) to receive dapagliflozin (10 mg once daily) or placebo. Exclusion criteria included type 1 diabetes, polycystic kidney disease, lupus nephritis, antineutrophil cytoplasmic antibody-associated vasculitis, recent immunosuppressive therapy for primary or secondary kidney disease, New York Heart Association class IV congestive heart failure, myocardial infarction, unstable angina, stroke or transient ischemic attacks, or recent coronary revascularization or valvular repair/replacement. All participants received a stable dose of renin-angiotensin system inhibitor for 4 weeks prior to screening, and the vast majority received a maximum tolerated dose at enrollment. Randomization was monitored to ensure that at least $30 \%$ of participants recruited did not have diabetes and that no more than $10 \%$ had stage 2 CKD. Participants were randomly assigned to receive dapagliflozin $(n=2152)$ or matching placebo $(n=2152)$ to ensure a 1:1 ratio of the 2 regimens. Dapagliflozin and placebo had identical appearance and administration schedules. All participants and trial

\section{Outcomes Research in Review SECTION EDITORS}

KatRINA F. MATEO, PHD, MPH

CUNY School of Public Health New York, NY

TAISHI HIRAI, MD

University of Missouri

Columbia, MO
DANIEL ISAAC, DO, MS

Michigan State University

East Lansing, Ml

WILLIAM HUNG, MD, MPH

Icahn School of Medicine at Mount Sinai

New York, NY
FRED Ko, MD, MS

Icahn School of Medicine at Mount Sinai New York, NY

GORDON NGAI, MD, MPH

Icahn School of Medicine at Mount Sinai New York, NY 
personnel (except members of the independent data monitoring committee) were unaware of the trial-group assignments. After randomization, in-person study visits were conducted at 2 weeks, at 2, 4, and 8 months, and at 4-month intervals thereafter.

Main outcome measures. The primary outcome was a composite of the first occurrence of either a sustained decline in the estimated GFR of at least $50 \%$, end-stage kidney disease, or death from renal or cardiovascular causes. Secondary outcomes, in hierarchical order, were: (1) the composite kidney outcome of a sustained decline in the estimated GFR of at least 50\%, end-stage kidney disease, or death from renal causes; (2) a composite cardiovascular outcome defined as hospitalization for heart failure or death from cardiovascular causes; and (3) death from any cause. All outcomes were assessed by time-toevent analyses.

Given the extensive prior experience with dapagliflozin, only selected adverse events were recorded. These included serious adverse events, adverse events resulting in the discontinuation of dapagliflozin or placebo, and adverse events of interest to dapagliflozin (eg, volume depletion symptoms, renal events, major hypoglycemia, fractures, diabetic ketoacidosis, events leading to higher risk of lower limb amputation, and lower limb amputations).

Main results. On March 26, 2020, the independent data monitoring committee recommended stopping the trial because of clear efficacy on the basis of 408 primary outcome events. The participants were $61.8 \pm 12.1$ years of age, and 1425 participants (33.1\%) were female. The baseline mean estimated GFR was $43.1 \pm 12.4$ $\mathrm{mL} / \mathrm{min} / 1.73 \mathrm{~m}^{2}$, the median urinary albumin-to-creatinine ratio was 949, and 2906 participants (67.5\%) had type 2 diabetes. Over a median of 2.4 years, a primary outcome event occurred in 197 participants (9.2\%) in the dapagliflozin group and 312 (14.5\%) in the placebo group (hazard ratio [HR], 0.61; 95\% confidence interval [Cl], 0.51-0.72; $P$ $<0.001)$. The number of participants who needed to be treated during the trial period to prevent 1 primary outcome event was 19 (95\% Cl, 15-27). The beneficial effect of dapagliflozin compared with placebo was consistent across all 8 prespecified subgroups (ie, age, sex, race, geographic region, type 2 diabetes, estimated GFR, urinary albumin-to-creatinine ratio, and systolic blood pressure) for the primary outcome. The effects of dapagliflozin were similar in participants with type 2 diabetes and in those without type 2 diabetes.

The incidence of each secondary outcome was similarly lower in the dapagliflozin-treated group than in the placebo group. The HR for the composite kidney outcome of a sustained decline in the estimated GFR of at least $50 \%$, end-stage kidney disease, or death from renal causes was 0.56 (95\% Cl, 0.45-0.68; $P<0.001)$, and the $\mathrm{HR}$ for the composite cardiovascular outcome of hospitalization for heart failure or death from cardiovascular causes was $0.71(95 \% \mathrm{Cl}, 0.55-0.92 ; P=0.009)$. Death occurred in 101 participants (4.7\%) in the dapagliflozin group and 146 participants (6.8\%) in the placebo group (HR, 0.69; 95\% Cl, 0.53-0.88; $P=0.004)$. The known safety profile of dapagliflozin was confirmed by the similar overall incidences of adverse events and serious adverse events in the dapagliflozin and placebo groups.

Conclusion. In patients with CKD, with or without type 2 diabetes, the risk of a composite of a sustained decline in the estimated GFR of at least 50\%, end-stage kidney disease, or death from renal or cardiovascular causes was significantly lowered by dapagliflozin treatment.

\section{Commentary}

Although SGLT2 inhibitors were designed to reduce plasma glucose and hemoglobin A1c (HbA1c) by increasing urinary glucose excretion in a non-insulin-dependent fashion, an increasing number of clinical trials have demonstrated their possible cardiovascular and renal benefits that extend beyond glycemic control. In 2008, the US Food and Drug Administration (FDA) issued a guidance recommending the evaluation of long-term cardiovascular outcomes prior to approval and commercialization of new antidiabetic therapies to ensure minimum cardiovascular risks following the discovery of cardiovascular safety issues associated with antidiabetic compounds, including rosiglitazone, after drug approval. No one foresaw that this recommendation would lead to the discovery of new classes of antidiabetic drugs (glucagon-like peptide 1 [GLP1] and SGLT2 inhibitors) that improve cardiovascular 


\section{Outcomes Research in Review}

outcomes. A series of clinical trials of SGLT2 inhibitors, including empagliflozin, ${ }^{1}$ canagliflozin, ${ }^{2}$ and dapagliflozin, ${ }^{3}$ showed a reduction in cardiovascular death and hospitalization due to heart failure among patients with type 2 diabetes. Furthermore, a meta-analysis from 2019 found that SGLT2 inhibitors reduced the risk of a composite of cardiovascular death or hospitalization for heart failure by $23 \%$ and the risk of progression of kidney failure by $45 \%$ in patients with diabetes. ${ }^{4}$ Thus, the strong and consistent evidence from these large and well-designed outcome trials led the American Diabetes Association in its most recent guidelines to recommend adding SGLT2 inhibitors to metformin for the treatment of patients with type 2 diabetes with or at high risk of atherosclerotic cardiovascular disease, heart failure, or CKD, regardless of baseline $\mathrm{HbA} 1 \mathrm{c}$ levels or $\mathrm{HbA1c}$ target. ${ }^{5}$ As a result of the compelling effects of SGLT2 inhibitors on cardiovascular outcomes in diabetic patients, as well as increasing evidence that these clinical effects were independent of glycemic control, several subsequent trials were conducted to evaluate whether this new class of drugs may improve clinical outcomes in nondiabetic patients.

The Dapagliflozin and Prevention of Adverse Outcomes in Heart Failure (DAPA-HF) was the first clinical trial to investigate the effect of SGLT2 inhibitors on cardiovascular disease in nondiabetic patients. Findings from DAPA-HF showed that dapagliflozin reduced the risk of worsening heart failure or death from cardiovascular causes, independent of the presence of underlying diabetes. This initial finding resonates with a growing body of evidence ${ }^{6,7}$ that supports the use of SGLT2 inhibitors as an adjunctive therapy for heart failure in the absence of diabetes.

The Canagliflozin and Renal Events in Diabetes with Established Nephropathy Clinical Evaluation (CREDENCE) trial showed that long-term administration of canagliflozin conferred cardiovascular, as well as renal, protection in patients with type 2 diabetes with CKD. ${ }^{8}$ Similar to the protective effects on heart failure, the renal benefits of SGLT2 inhibitors appeared to be independent of their blood glucose-lowering effects. Thus, these recent discoveries led to the design of the DAPA-CKD trial to further assess the long-term efficacy and safety of the SGLT2 inhibitor dapagliflozin in patients with CKD precipitated by causes other than type 2 diabetes. Although diabetes is the most common cause for CKD, it nonetheless only accounts for $40 \%$ of all CKD etiologies. To date, the only classes of medication that have been shown to slow a decline in kidney function in patients with diabetes are angiotensin-converting enzyme (ACE) inhibitors and angiotensin receptor blockers (ARBs). Given that CKD is an important contributor to illness, is associated with diminished quality of life and reduced life expectancy, and increases health care costs, the findings of the DAPACKD trial are particularly significant as they show a renal benefit of dapagliflozin treatment across CKD stages that is independent of underlying diabetes. Therefore, SGLT2 inhibitors may offer a new and unique treatment option for millions of patients with CKD worldwide for whom ACE inhibitors and ARBs were otherwise the only treatments to prevent kidney failure. Moreover, with a number-needed-to-treat of 19 to prevent 1 composite renal vascular event over a period of 2.4 years, dapagliflozin requires a much lower number needed to treat compared to ACE inhibitors and ARBs in similar patients.

The trial has several limitations in study design. For example, the management of diabetes and hypertension were left to the discretion of each trial site, in keeping with local clinical practice and guidelines. It is unknown whether this variability in the management of comorbidities that impact kidney function had an effect on the study's results. In addition, the trial was stopped early as a result of recommendations from an independent committee due to the demonstrated efficacy of dapagliflozin. This may have reduced the statistical power to assess some of the secondary outcomes. Finally, the authors discussed an initial dip in the estimated GFR after initiation of dapagliflozin treatment, similar to that observed in other SGLT2 inhibitor clinical trials. However, they were unable to ascertain the reversibility of this effect after the discontinuation of dapagliflozin because assessment of GFR was not completed after trial closure. Nonetheless, the authors specified that the reversibility of this initial estimated GFR dip had been assessed and observed in other clinical trials involving dapagliflozin.

The nonglycemic benefits of SGLT2 inhibitors, including improvement in renal outcomes, have strong implications for the future management of patients with 
CKD. If this indication is approved by the FDA and recommended by clinical guidelines, the ease of SGLT2 inhibitor prescription (eg, minimal drug-drug interaction, no titration), treatment administration (orally once daily), and safety profile may lead to wide use of SGLT2 inhibitors by generalists, nephrologists, and endocrinologists in preserving or improving renal outcomes in patients at risk for end-stage kidney disease. Given that SGLT2 inhibitors are a new class of pharmacologic therapeutics, patient education should include a discussion of the possible side effects, such as euglycemic ketoacidosis, genital and urinary tract infection, and foot and leg amputation. Finally, as Strandberg and colleagues reported in a recent commentary, ${ }^{9}$ the safety of SGLT2 inhibitors in older adults with multimorbidity, frailty, and polypharmacy remains unclear. Thus, future studies of SGLT2 inhibitors are needed to better evaluate their clinical effects in older adults.

\section{Applications for Clinical Practice}

This trial enrolled a dedicated patient population with CKD and demonstrated a benefit of dapagliflozin in reducing renal and cardiovascular outcomes, regardless of baseline diabetes status. These drugs (dapagliflozin as well as other SGLT2 inhibitors) will likely have a prominent role in future CKD management guidelines. Until then, several barriers remain before SGLT2 inhibitors can be widely used in clinical practice. Among these barriers are FDA approval for their use in patients with and without diabetes with an estimated GFR $<30 \mathrm{~mL} / \mathrm{min} / 1.73 \mathrm{~m}^{2}$ and lowering the costs of this class of drugs.

-Rachel Litke, MD, PhD

Icahn School of Medicine at Mount Sinai

Fred Ko, MD, MS

doi:10.12788/jcom.0030

\section{References}

1. Zinman B, Wanner C, Lachin JM, et al. Empagliflozin, cardiovascular outcomes, and mortality in type 2 diabetes. $N$ Engl J Med. 2015;373:2117-2128.

2. Neal B, Perkovic V, Matthews DR. Canagliflozin and cardiovascular and renal events in type 2 diabetes. N Eng/ J Med. 2017;377:2099.

3. Wiviott SD, Raz I, Bonaca MP, et al. Dapagliflozin and cardiovascular outcomes in type 2 diabetes. N Engl J Med. 2019;380:347-357.

4. Zelniker TA, Wiviott SD, Raz I, Sabatine MS. SGLT-2 inhibitors for people with type 2 diabetes - Authors' reply. Lancet. 2019;394:560-561.

5. American Diabetes Association 10. Cardiovascular disease and risk management: standards of medical care in diabetes-2020. Diabetes Care. 2020;43(Suppl 1):S111-S34.

6. Packer M, Anker SD, Butler J, et al. Cardiovascular and renal outcomes with empagliflozin in heart failure. $N$ Engl J Med. 2020;383:1413-1424.

7. Zannad F, Ferreira JP, Pocock SJ, et al. SGLT2 inhibitors in patients with heart failure with reduced ejection fraction: a meta-analysis of the EMPEROR-Reduced and DAPA-HF trials. Lancet. 2020; 396:819-829.

8. Perkovic V, Jardine MJ, Neal B, et al. Canagliflozin and renal outcomes in type 2 diabetes and nephropathy. $N$ Engl $J$ Med. 2019;380:2295-2306.

9. Strandberg TE, Petrovic M, Benetos A. SGLT-2 inhibitors for people with type 2 diabetes. Lancet. 2019;394:560.

\section{Cognitive Behavioral Therapy Plus Placebo Is Inferior to NSAID Therapy for Arthritis Pain}

Fraenkel L, Buta E, Suter L, et al. Nonsteroidal anti-inflammatory drugs vs cognitive behavioral therapy for arthritis pain a randomized withdrawal trial. JAMA Intern Med. 2020;180:1194-1202.

\section{Study Overview}

objective. To examine whether discontinuation of nonsteroidal anti-inflammatory drug (NSAID) therapy and initiation of telephone-based cognitive behavioral therapy (CBT) is not worse than continuation of NSAIDs in the management of arthritis pain.
Design. Randomized controlled trial with noninferiority design.

Setting and participants. This study was a multicenter trial conducted across 4 Veterans Affairs health care systems in Boston, Providence, Connecticut, and North Florida/ 


\section{Outcomes Research in Review}

South Georgia that started September 2013 and ended September 2018. Eligibility criteria included being age 20 years or older, radiographic evidence of knee osteoarthritis, and use of an NSAID for knee pain on most days of the month for at least the past 3 months. Exclusion criteria included significant hearing impairments that may impede the conduct of the trial, current opioid prescriptions excluding tramadol, contraindications to NSAID use, recent or scheduled intra-articular injections or surgery, comorbid conditions other than knee pain that limited walking, and bilateral knee replacements or pain only in the replaced knee. Concurrent use of tramadol and other non-NSAID analgesics was permitted.

A total of 490 participants took part in the 2-week run-in period where their NSAID regimen was discontinued and they were started on a standardized dose of the NSAID meloxicam $15 \mathrm{mg}$ daily. During the run-in period, 126 participants were excluded for several reasons, including worsening pain and patient withdrawal, yielding 364 participants who were randomized to continue meloxicam treatment or placebo for 4 weeks with blinding.

Intervention. Subsequent to the 4-week phase 1 placebo controlled trial, participants in the placebo group were given CBT via telephone (unblinded) for 10 weeks, and the meloxicam group continued treatment with meloxicam for phase 2. The CBT group received 10 modules over 10 weeks in 30- to 45-minute telephone contacts with a psychologist using a treatment manual modified for knee osteoarthritis. These modules consisted of 1 introductory module, 8 pain coping skills modules (eg, deep breathing and visual imagery, progressive muscle relaxation, physical activity and bodily mechanics, identifying unhealthy thoughts, balancing unhealthy thoughts, managing stress, time-based pacing, and sleep hygiene), and a final module emphasizing skill consolidation and relapse prevention. Outcomes were assessed at the end of the phase 1 and phase 2 periods.

Main outcome measures. Main study outcome measures included pain as measured with the Western Ontario and McMaster Universities Osteoarthritis Index (WOMAC) at 4 weeks. Secondary outcomes included the WOMAC pain score, disability score, and global impression of change after treatment at 14 weeks. The WOMAC pain scale ranges from 0 to 20, and consists of 5 questions regarding severity of pain during walking, stair use, lying in bed at night, sitting, and standing, with 0 indicating no pain; 1, mild pain; 2, moderate pain; 3, severe pain; and 4, very severe pain for each item. The WOMAC disability scale measures self-reported difficulty in performing tasks that reflect lower-extremity physical function, including climbing stairs, rising from a chair, walking, and other activities of daily living. The global impression of change after treatment was measured on a 5-point scale (where 1 indicates much better and 5 indicates much worse). The minimum clinically important difference of the WOMAC pain scale is 2 , based on prior literature. With the noninferiority design, the margin was set as a score of 1 .

Main results. The placebo group consisted of 180 participants, with an average age of 58.2 years (SD, 11.8 years); $89 \%$ of them were male. The meloxicam group consisted of 184 participants, with an average age of 58.6 years (SD, 10 years); $84 \%$ of them were male. The average body mass index was 33.9 and 33.4 in each group, respectively. For the primary outcome, the placebo group had a worse pain score than the meloxicam group at 4 weeks (difference of 1.4; 95\% confidence interval, 0.82.0). At 14 weeks, the placebo group (with CBT) had a worse pain score than the meloxicam group (difference of $0.8 ; 95 \% \mathrm{Cl}, 0.2-1.4$ ). There was no statistically significant difference in the disability score or global impression of change after treatment score between the 2 groups. The observed difference in pain score did not, however, exceed the minimum clinically important difference.

Conclusion. Placebo treatment and CBT are inferior to NSAIDs in managing pain for patients with knee osteoarthritis. The difference in pain may not be clinically important, and there were no differences in function at 14 weeks.

\section{Commentary}

Osteoarthritis is a common chronic condition that causes pain and disability and is often treated with oral analgesics. NSAIDs, despite few high-quality trials demonstrating their efficacy, are among the most commonly used 
treatment for osteoarthritis pain.' NSAID therapy, however, does have potential side effects, such as gastric reflux and renal dysfunction. ${ }^{2}$ This withdrawal trial with placebo control contributes further evidence of the effectiveness of NSAIDs on knee osteoarthritis, demonstrating that indeed NSAIDs improve pain scores to a greater degree than placebo treatment. Augmenting placebo treatment with nonpharmacologic CBT was inferior to NSAIDs in pain management. The authors pointed out that the difference in pain score may not be clinically important, and that lower-extremity function was not different between the groups, concluding that, despite the higher pain score, CBT could be a treatment option, particularly for those who may have difficulty tolerating NSAID treatment.

The study population had a number of chronic conditions in addition to having knee arthritis, and thus likely were taking multiple medications for chronic disease management. Use of multiple medications is associated with an increased risk of rug interactions and adverse effects of medications. ${ }^{3}$ Thus, this attempt to assess whether a nonpharmacologic alternative treatment is noninferior to a drug treatment is a step toward building the evidence base for deprescribing and enhancing medication safety. ${ }^{4}$ Previous studies have examined other nonpharmacologic treatments for knee arthritis, such as acupuncture, ${ }^{5}$ and it is worthwhile to consider combining nonpharmacological approaches as an alternative to oral analgesic medication use.

\section{Applications for Clinical Practice}

This study advances our understanding of the effect of NSAID use on knee osteoarthritis when compared to placebo with CBT. Although this is a negative study that failed to show that placebo combined with CBT is noninferior to NSAIDs, it did quantify the expected treatment effect of NSAIDs and showed that this effect likely is not clinically important and/or does not alter lowerextremity function. Further studies are needed to identify other nonpharmacologic approaches and test whether combinations of approaches are effective in the management of chronic pain from osteoarthritis.

-William W. Hung, MD, MPH doi:10.12788/jcom.0029

\section{References}

1. Wongrakpanich S, Wongrakpanich A, Melhado K, Rangaswami J. A comprehensive review of non-steroidal anti-inflammatory drug use in the elderly. Aging Dis. 2018;9:143-150.

2. Pilotto A, Franceschi M, Leandro G, Di Mario F. NSAID and aspirin use by the elderly in general practice: effect on gastrointestinal symptoms and therapies. Drugs Aging. 2003;20:701-710.

3. Steinman MA. Polypharmacy-time to get beyond numbers. JAMA Intern Med. 2016;176:482-483.

4. Rashid R, Chang C, Niu F, et al. Evaluation of a pharmacist-managed nonsteroidal anti-inflammatory drugs deprescribing program in an integrated health care system. J Manag Care Spec Pharm. 2020;26:918-924.

5. Sun J, Zhao Y, Zhu R, et al. Acupotomy therapy for knee osteoarthritis pain: systematic review and meta-analysis. Evid Based Complement Alternat Med. 2020;2020:2168283.

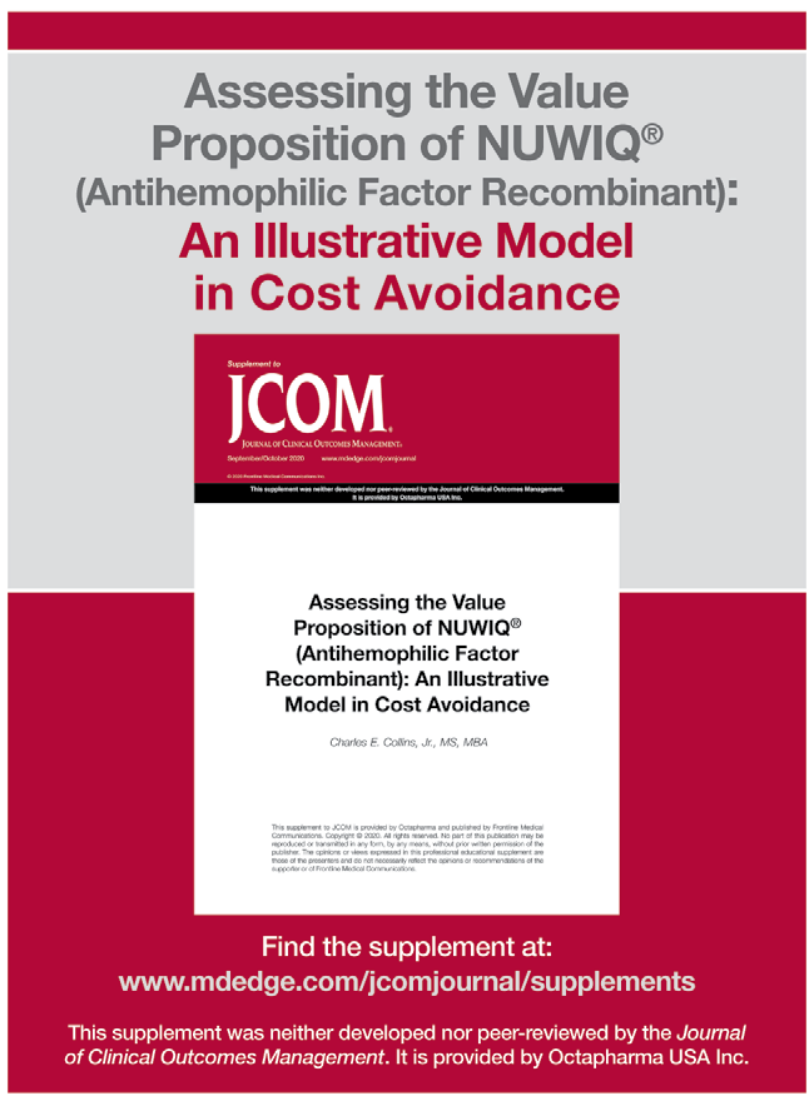

